\title{
Reduction in Cylindrical Grasp Strength Is Associated With Early Thumb Carpometacarpal Osteoarthritis
}

\author{
Monica J. Coughlan BS, Alexandra Bourdillon, Joseph J. Crisco PhD, \\ Deborah Kenney MS, OTR, Arnold-Peter Weiss MD, Amy L. Ladd MD
}

Received: 24 July 2016/ Accepted: 26 October 2016/Published online: 7 November 2016

(C) The Association of Bone and Joint Surgeons $\mathbb{R} 2016$

\begin{abstract}
Background Advanced thumb carpometacarpal (CMC) osteoarthritis (OA) can cause substantial impairment in hand function, from grasping heavy objects to fine manipulation of implements and tools. In the clinical setting, we commonly measure the grip strength of gross grasp with a hand dynamometer in patients with CMC OA. Cylindrical grasp, which requires more thumb contribution than gross grasp, is an alternative method of measuring grip strength and one that may provide insight into thumb-
\end{abstract}

One of the authors (MJC) has received research support funding from a Stanford University MedScholars grant. Both institutions of the authors (MJC, AB, JJC, DK, A-PW, ALL) have received funding from the National Institute of Arthritis and Musculoskeletal and Skin Diseases of the National Institutes of Health under Award Number R01 AR059185.

All ICMJE Conflict of Interest Forms for authors and Clinical Orthopaedics and Related Research ${ }^{\circledR}$ editors and board members are on file with the publication and can be viewed on request.

Clinical Orthopaedics and Related Research ${ }^{\mathbb{R}}$ neither advocates nor endorses the use of any treatment, drug, or device. Readers are encouraged to always seek additional information, including FDAapproval status, of any drug or device prior to clinical use.

Each author certifies that his or her institution approved the human protocol for this investigation, that all investigations were conducted in conformity with ethical principles of research, and that informed consent for participation in the study was obtained.

The content is solely the responsibility of the authors and does not necessarily represent the official views of the National Institutes of Health.

This work was performed at Stanford University, Stanford, CA, USA, and Brown University, Providence, RI, USA.

M. J. Coughlan ( $₫)$, A. Bourdillon, D. Kenney, A. L. Ladd Department of Orthopaedic Surgery, Robert A. Chase Hand \& Upper Limb Center, Stanford University, 450 Broadway Street, Pavilion A, 2nd Floor, Dept A26 MC 6342, Redwood City, CA 94063, USA

e-mail: mcoughlan@stanford.edu related conditions. Because gross grasp and cylindrical grasp use the thumb in different planes, measurement of gross grasp alone might underestimate impairment. Therefore, it is important to evaluate cylindrical grasp as well. To our knowledge this tool has yet to be examined in a population with early thumb CMC OA.

Questions/Purposes (1) Is cylindrical grasp and gross grasp strength reduced in subjects with early thumb CMC OA compared with asymptomatic control subjects? (2) What is the association of cylindrical and gross grasp to thumb CMC OA after adjusting for age, sex, and hand dominance?

Methods We recruited 90 subjects with early symptomatic and radiographic thumb CMC OA and 38 asymptomatic healthy control subjects for this multisite controlled study. Demographic information, hand examination, comprehensive histories, plain film radiographs, and cylindrical and gross grasp strength data were collected on all 128 subjects. Mean grasp strength was calculated for cylindrical and gross grasp in the population with early CMC OA and the control population. A t-test was performed on cylindrical and gross grasp to evaluate the difference between the mean in the control and early CMC OA populations. We used separate linear regression models for the two types of grasp to further quantify the association of grasp with a diagnosis of early thumb CMC OA controlling for age, sex, and whether the subject used their dominant or nondominant hand in the study.

\section{J. J. Crisco, A.-P. Weiss}

Department of Orthopaedics, Rhode Island Hospital, The Warren Alpert Medical School of Brown University, Providence, RI, USA 
Results Cylindrical grasp was weaker in the population with thumb CMC OA compared with healthy control subjects $(6.3 \pm 2.7 \mathrm{~kg}$ versus $8.4 \pm 2.5 \mathrm{~kg}$; mean difference, $2.1 ; 95 \% \mathrm{CI}, 1.1-3.1 ; \mathrm{p}<0.001)$, but there was no difference in gross grasp force $(29.6 \pm 11.6 \mathrm{~kg}$ versus 31.4 $\pm 10.1 \mathrm{~kg}$; mean difference, $1.7 ; 95 \% \mathrm{CI},-2.5$ to $6.0 ; \mathrm{p}=$ $0.425)$. When adjusting for age, sex, and handedness, cylindrical grasp reduction was related to CMC OA $(\beta=$ -2.3; standard error [SE], 0.46; $\mathrm{p}<0.001)$ (Y-intercept $=$ 8.2; $\left.\mathrm{SE}, 1.8 ; \mathrm{R}^{2}=0.29\right)$, whereas gross grasp was not reduced in early thumb CMC OA $(\beta=-2.8$; SE, $1.6 ; \mathrm{p}=$ 0.072) (Y-intercept $=34.3 ; \mathrm{SE}, 6.3 ; \mathrm{R}^{2}=0.48$ ).

Conclusions A reduction in cylindrical grasp is associated with early symptomatic and radiographic CMC OA, whereas gross grasp is not associated with early thumb CMC OA, suggesting that cylindrical grasp may be a better tool to detect changes in thumb and hand function seen during early disease stages.

Clinical Relevance Cylindrical grasp may serve as a more-sensitive measure for detecting early changes in early CMC OA. The associated decline in hand function also might provide an opportunity for measuring the effectiveness of treatment and intervention.

\section{Introduction}

Thumb carpometacarpal (CMC) osteoarthritis (OA) is a common debilitating joint disorder, affecting approximately $20 \%$ to $65 \%$ of adults $[15,22]$. Patients with thumb CMC OA report reduced function with routine hand use involving the thumb $[1,5,11,12,19]$. Pain appears to contribute to the diminished function and decreased hand strength observed in patients with CMC OA [12], but mechanical dysfunction arising from joint deformity also might play a large role [16, 24]. Grip strength is a gross measure of functional impairment [19]. It often is reported in studies of thumb CMC OA [1, 5, 11-13, 25] and commonly is used in the clinic to evaluate hand function. Decreases in grip strength are observed in patients with advanced thumb $\mathrm{CMC} \mathrm{OA}$, potentially corresponding to other biomechanical changes at the thumb CMC joint as the disease progresses [1, 5, 7, 10, 13, 17, 24, 28]. One method of measuring grip strength is with a dynamometer. As a result of their shape and positioning, dynamometers do not measure the forces on the thumb during gross grasp. The thumb contributes the least of all digits to grip strength measurement [2]. Other forms of grasp have been evaluated with cylindrical grasp reported as an alternative measure for grip strength [27]. During cylindrical grasp, the thumb is in a position of palmar abduction and opposes the combined forces of the other four fingers, which have been found to produce up to $120 \mathrm{~kg}$ of compressive forces on the CMC joint in a cadaver biomechanical study [4].
Additionally, cylindrical grasp is the most common type of grasp used during functional activities [23]. Cylindrical grasp has been used to investigate stability of the $\mathrm{CMC}$ joint in early $\mathrm{CMC} \mathrm{OA}$, and it was found that there are no changes in stability of the thumb CMC joint in this population during cylindrical grasp [8].

However, to our knowledge, only one study on functional and biomechanical changes in early thumb CMC OA exists, and it does not evaluate grip strength [18]. Although data exist regarding gross grasp in many populations, including a healthy population and a population with advanced thumb CMC OA [1, 5, 13, 17, 20, 25, 26], there have been no studies that examine this grip strength in a population with early thumb CMC OA. Cylindrical grasp has been investigated only as a tool to examine thumb CMC OA joint stability, and has not yet been explored as a grip strength measurement [7, 8, 16]. Additionally, to our knowledge, there has been no direct comparison of gross grasp with cylindrical grasp strength in patients with early $\mathrm{CMC} \mathrm{OA}$. As the thumb plays a different role in gross and cylindrical grasp, it is likely that these two tools may detect different changes in the thumb CMC joint. With the high compressive forces that the thumb joint experiences in cylindrical grasp [4], we would suspect that this tool may detect changes in hand function or pain earlier than gross grasp. With gross grasp as the current gold standard for assessing grip strength across the spectrum of thumb CMC $\mathrm{OA}$, it is important to compare the two measurements to determine whether cylindrical grasp may be a better tool at early disease stages.

We therefore asked: (1) Is cylindrical grasp and gross grasp strength reduced in subjects with early thumb CMC OA compared with asymptomatic control subjects? (2) What is the association of cylindrical and gross grasp to CMC OA after adjusting for age, sex, and hand dominance?

\section{Patients and Methods}

Symptomatic subjects with early thumb CMC OA and healthy control subjects were recruited for this multisite controlled study. A power analysis based on normative data of gross grasp in a 55- to 59-year-old right-hand dominant population was conducted and determined that a minimum of 24 subjects was necessary to detect a $20 \%$ reduction in grasp in the population with early thumb CMC OA [17]. A total of 128 subjects were identified through a larger ongoing National Institutes of Health investigation at two study sites (90 subjects with early thumb CMC OA and 38 healthy control subjects). An institutional review board at both study sites provided ethical approval of the study before patient enrollment began. Informed consent was 
obtained from all participants, and a hand examination with a detailed history including hand dominance, age, and sex was recorded for each subject. Plain film radiographs (posteroanterior, lateral, stress view, and Robert's view of the thumb) also were obtained to evaluate for inclusion and exclusion criteria.

Early thumb CMC OA was defined as having absent or early radiographic change (modified Eaton Stage 0-I) accompanied by thumb pain or discomfort [14]. All subjects were evaluated by a board certified hand surgeon to confirm a diagnosis of early thumb CMC OA, and exclude other possible disorders for thumb pain or discomfort. Inclusion criteria for the healthy population were defined as having no pain in the thumb and no radiographic evidence (modified Eaton Stage 0) of thumb CMC OA. Exclusion criteria for both populations included a diagnosis of other causes of base of thumb pain (trigger thumb, DeQuervain's tenosynovitis, scaphotrapezotrapezoidal arthritis), inflammatory arthritis, connective tissue or metabolic bone disease, and history of fracture, ligament injury, or surgery of the ipsilateral hand or wrist.

An instrumented digital dynamometer (Greenleaf Medical Systems, Palo Alto, CA, USA) was used to assess gross grasp following the American Society of Hand Therapists guidelines (Fig. 1) [21]. The dynamometer was set at the hand position 3 setting throughout the study and was not adjusted for patient hand size. Each subject completed three maximum force trials with 45 seconds of rest separating each trial. The peak force from each trial was recorded. The three peak forces were averaged to obtain an average maximum force for each subject.

The cylindrical grasp tool was designed based on prior studies [3, 8, 9]. A custom-made polycarbonate cylinder (66 $\mathrm{mm}$ diameter, $117 \mathrm{~mm}$ height) with a single axis load cell (0-50 pounds [0-22.7 kg], Model D Thru-Hole Load Cell; Honeywell International Inc, Morristown, NJ, USA) recessed in the side of the cylinder and covered by a concave button (overall diameter of $67.5 \mathrm{~mm}$ ) was used to measure cylindrical grasp [3,9]. The force generated by the distal phalanx of the thumb was measured perpendicular to the surface of the button. Each subject's hand and wrist were positioned following a previously described protocol (Fig. 2) [9]. Each subject completed two maximum effort trials with 45 seconds of rest separating the trials, and the two peak forces were averaged to obtain an average maximum force for each subject. Data were collected using a custom-written LabVIEW (National Instruments, Austin, TX, USA) data acquisition and display program.

Summary demographic data were compared between the symptomatic thumb CMC OA group and the healthy control subjects (Table 1). The average age of the control group was 57 years, and the average age of the OA group

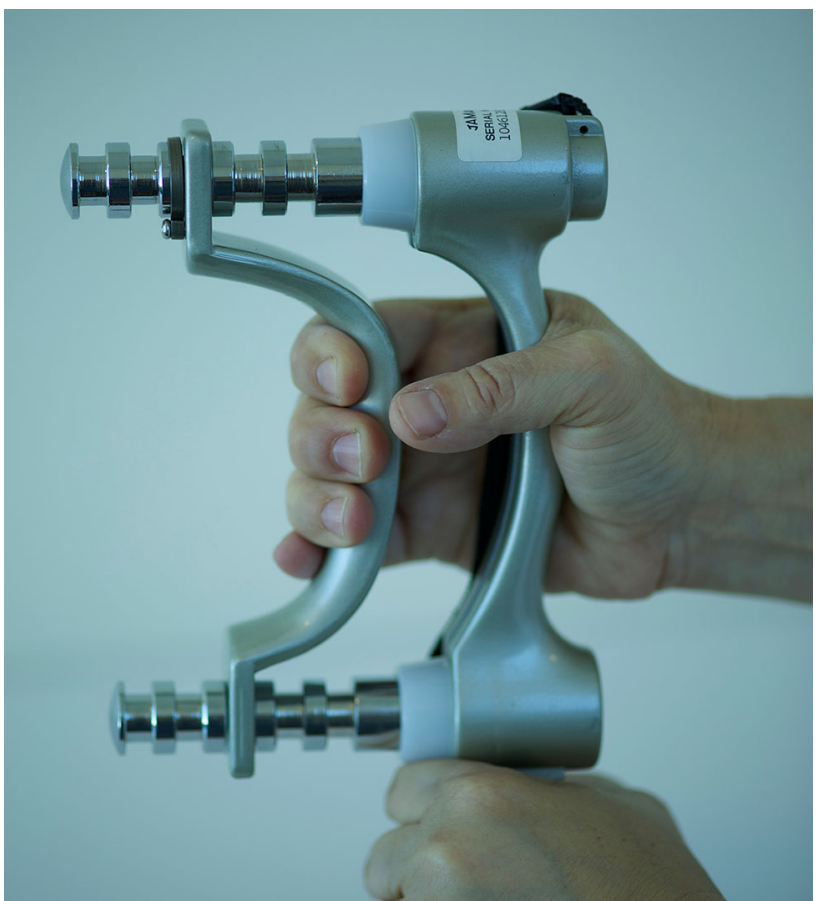

Fig. 1 Standard hand positioning for the instrumented digital dynamometer followed the American Society of Hand Therapists guidelines [21].

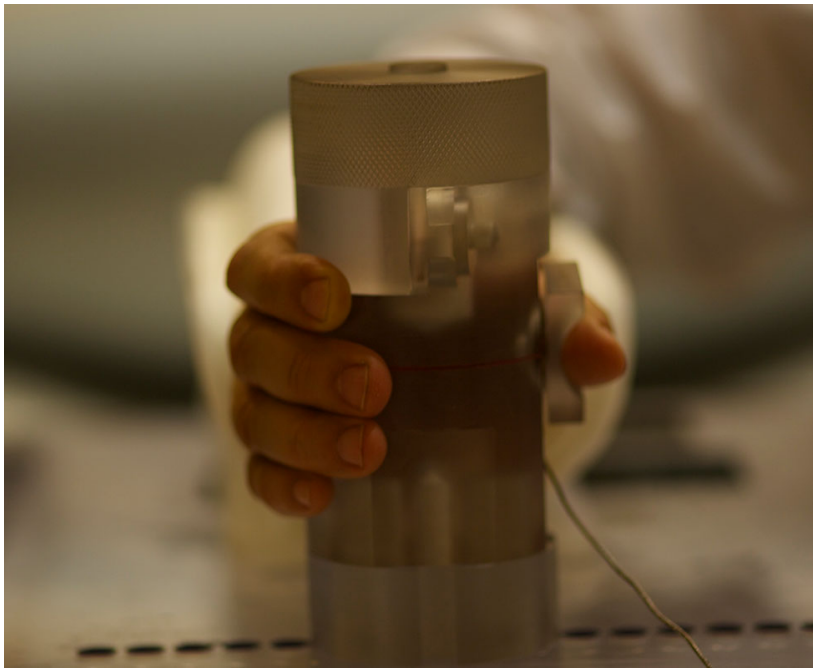

Fig. 2 Standard hand positioning for the cylindrical jig followed protocols outlined in a prior study [9].

was 57 years. There was no difference in the proportion of men $(39 \%$ [15/38] in the control group, 47\% [42/90] in the early CMC OA group; $\mathrm{p}=0.410$ ) or the proportion of subjects who used their dominant hand for the force measurements in the two groups $(68 \%$ [26/38] in the control group, 63\% [57/90] in the early CMC OA group; $\mathrm{p}=$ $0.642)$. 
Table 1. Demographic information

\begin{tabular}{llll}
\hline Variable & Subjects with thumb CMC OA, mean (SD), $\mathrm{n}=90$ & Control subjects, mean $(\mathrm{SD}), \mathrm{n}=38$ & $\mathrm{p}$ Value \\
\hline Age (years) & $57(7.3)$ & $57(7.7)$ & 0.728 \\
Male sex (\%) & $47(50.2)$ & $39(49.5)$ & 0.410 \\
Dominant hand is study hand $(\%)$ & $63(48.5)$ & $68(47.1)$ & 0.642 \\
\hline
\end{tabular}

$\mathrm{CMC} \mathrm{OA}=$ carpometacarpal osteoarthritis.

Table 2. Mean maximum grasp values and differences between the mean

\begin{tabular}{llclrr}
\hline Variable & $\begin{array}{l}\text { Subjects with thumb CMC OA } \\
\text { mean (SD), } \mathrm{n}=90\end{array}$ & $\begin{array}{l}\text { Control subjects, } \\
\text { mean (SD), } \mathrm{n}=38\end{array}$ & $\begin{array}{l}\text { Mean difference } \\
(95 \% \text { CI) }\end{array}$ & $\mathrm{t}$ Value & $\mathrm{p}$ Value \\
\hline Cylindrical grasp (kg) & $6.3(2.7)$ & $8.4(2.5)$ & $2.1(1.1$ to 3.1$)$ & 4.1 \\
Gross grasp (kg) & $29.6(11.6)$ & $31.4(10.1)$ & $1.7(-2.5$ to 6.0$)$ & 0.8 & 0.425 \\
\hline
\end{tabular}

CMC OA = carpometacarpal osteoarthritis.

\section{Statistical Analysis}

Data were analyzed using SAS ${ }^{\circledR}$ (SAS Institute, Cary, NC, USA). Means and standard deviations for gross grasp and cylindrical grasp were calculated for both populations. Ttests were performed on the cylindrical and gross grasp data to compare the difference between means for the control and early CMC OA populations. Adjusted linear regression was performed for each type of grasp with the grasp force measurement as the dependent variable and OA status as the independent variable controlling for age, sex, and whether the subject was using their dominant or nondominant hand. Results were reported as parameter estimates with standard errors, and $\mathrm{p}$ values. Adjusted $\mathrm{R}^{2}$ values also were reported for each regression line. A $p$ value less than 0.050 was considered significant.

\section{Results}

Cylindrical grasp was weaker in the population with thumb CMC OA compared with healthy control subjects $(6.3 \pm$ $2.7 \mathrm{~kg}$ versus $8.4 \pm 2.5 \mathrm{~kg}$; mean difference, $2.1 \mathrm{~kg}$; $95 \%$ CI, $1.1-3.1 \mathrm{~kg} ; \mathrm{p}<0.001$ ), but there was no difference in gross grasp force $(29.6 \pm 11.6 \mathrm{~kg}$ versus $31.4 \pm 10.1 \mathrm{~kg}$; mean difference, $1.7 \mathrm{~kg}$; $95 \% \mathrm{CI},-2.5$ to $6.0 \mathrm{~kg}$; $\mathrm{p}=$ 0.425 ) (Table 2).

After adjusting for age, sex, and whether the dominant or nondominant hand was used for data collection, the linear regression model showed that radiographic and symptomatic thumb CMC OA was associated with weaker cylindrical grasp $(\beta=-2.3$; standard error $[\mathrm{SE}], 0.46 ; \mathrm{p}<$ $0.001)\left(\mathrm{Y}\right.$-intercept $\left.=8.2 ; \mathrm{SE}, 1.8 ; \mathrm{R}^{2}=0.29\right)($ Table 3$)$.
After adjusting for age, sex, and whether the dominant or nondominant hand was used for data collection, the linear regression model for gross grasp showed there is no association between early radiographic and symptomatic thumb CMC OA and gross grasp $(\beta=-2.8 ; \mathrm{SE}, 1.6 ; \mathrm{p}=0.072)$ $\left(\mathrm{Y}\right.$-intercept $\left.=34.3 ; \mathrm{SE}, 6.3 ; \mathrm{R}^{2}=0.48\right)($ Table 4$)$.

\section{Discussion}

Evaluation of grip strength is an important clinical assessment in subjects with thumb CMC OA to evaluate changes in hand function seen in the disease. Although gross grasp is the current tool used clinically, the thumb plays a minimal role during this type of grasp, whereas in cylindrical grasp the thumb plays a critical role, undergoing larger compressive forces during the task. Because of different thumb positioning in the two measurements, we considered that OA will have different effects on each grasp. Although is it known that gross grasp is reduced in subjects with advanced thumb CMC OA, to our knowledge, there have been no studies examining the utility of this tool or others during early disease stages. The purpose of our study was to evaluate cylindrical and gross grasp in a population with early thumb CMC OA compared with a control population, because earlier studies have focused primarily on later disease stages when examining the effect of hand OA on grip strength $[1,5,13]$. We found that a diagnosis of early thumb CMC OA was associated with weaker cylindrical grasp, whereas there was no association between early thumb CMC OA and gross grasp.

This study had several limitations. The cylindrical tool in this study had a fixed diameter, which was selected from 
Table 3. The relationship between cylindrical grasp and early CMC $\mathrm{OA}$

\begin{tabular}{lc}
\hline Variable & $\beta$ (standard error; $\mathrm{p}$ value $)$ \\
\hline CMC OA diagnosis & $-2.3(0.46 ;<0.001)$ \\
Male gender & $2.4(0.47 ;<0.001)$ \\
Age & $-0.02(0.03 ; 0.465)$ \\
Dominant hand & $0.73(0.44 ; 0.101)$ \\
Y intercept & $8.2(1.8 ;<0.001)$ \\
\hline
\end{tabular}

$\mathrm{CMC} \mathrm{OA}=$ carpometacarpal osteoarthritis.

Table 4. Relationship between gross grasp and early CMC OA

\begin{tabular}{lc}
\hline Variable & $\mathrm{B}($ standard error; $\mathrm{p}$ value $)$ \\
\hline CMC OA diagnosis & $-2.8(1.6 ; 0.072)$ \\
Male gender & $16.2(1.6 ;<0.001)$ \\
Age & $-0.20(0.12 ; 0.062)$ \\
Dominant hand & $3.1(1.5 ; 0.041)$ \\
Y Intercept & $34.3(6.3 ;<0.001)$
\end{tabular}

CMC OA = carpometacarpal osteoarthritis.

prior studies, thereby making it impossible to adjust the size of the tool to optimize grasp strength measurements for each subject. Furthermore, grip strength testing is only one of many potential measurements for evaluation of thumb CMC OA, not a standalone in the diagnosis or clinical decision-making regarding treatment of thumb CMC OA. We examined grip strength at a specific time in disease for our subjects with early thumb CMC OA compared with control subjects. Although quantification of grip strength at the subjects' baseline is useful to establish an understanding of how these tools may detect differences in grip strength at a specific time in the disease, its utility in measuring disease progression and further functional decline is unknown. Additionally, although the dynamometer has been described as a reliable grip strength measurement $[17,26]$, the cylindrical tool has not been as comprehensively studied, requiring further validation of its reliability as a tool before widespread clinical use [27]. Although this tool has not been adopted clinically, there are efforts to introduce similar cylindrical grasp tools for assessment of hand strength in musculoskeletal disease [27], and therefore studies such as the current one are needed to better understand the potential role of cylindrical grasp.

Cylindrical grasp is affected by early thumb OA, but gross grasp is not. Villafañe et al. [25] defined the minimum clinical important difference of grip strength when measured by a dynamometer between healthy subjects and subjects with late-stage thumb CMC OA to be $0.84 \mathrm{~kg}$ to $1.12 \mathrm{~kg}$ and found that healthy subjects had approximately

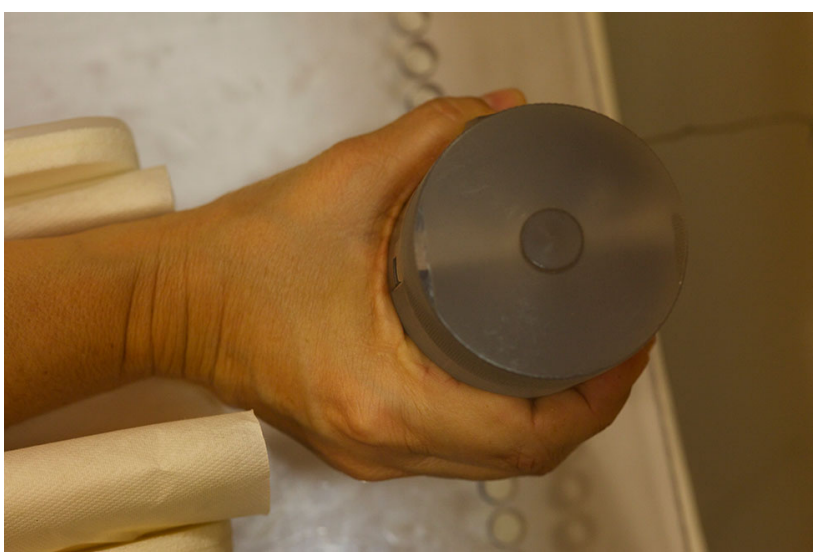

Fig. 3 Hand positioning for the cylindrical jig involves the thumb in a position of palmar abduction.

$50 \%$ greater grip strength compared with their counterparts with OA. We did not find as large a difference between the two groups in gross grasp or cylindrical grasp; however, the population with $\mathrm{OA}$ in the aforementioned study included subjects with more severe disease (Eaton Stages III-IV OA), whereas we restricted our study population to early disease (modified Eaton Stages 0-I). This suggests that although the dynamometer is a useful tool in later stages of disease, it may not be as useful during early disease stages, but conversely cylindrical grasp may be a useful tool during early disease. Hand positioning during cylindrical grasp may play an important role in thumb kinetics because the thumb is in a position of palmar abduction during cylindrical grasp, allowing it to oppose the forces of the long fingers (Fig. 3) [6]. Patients with thumb CMC OA often have difficulty with thumb abduction as a result of pain, and in advanced disease, the pain may become so severe that patients try to eliminate abduction from their activities of daily life. This continual avoidance of abduction results in secondary deformities of the joint, including adduction contractures of the first web space [22]. This discomfort with abduction often is seen early in the disease, which could contribute to the significant reduction in cylindrical grasp compared with that of a healthy population.

Our results further suggest that perhaps cylindrical grasp is a more sensitive test in measuring the decrease in forces during early disease compared with the standard clinical tool of gross grasp with a dynamometer. CMC joints affected by early OA do not yet show signs of instability during functional tasks; however, a decrease in key pinch strength has been documented in the same early stage of disease [8, 18]. Our results complement these findings, suggesting that although there may not be changes in joint stability at early disease stages, there may be changes in loading of the thumb that are detectable with certain clinical tools. 
In assessing dysfunction in early thumb CMC OA, cylindrical grasp strength measurements are more appropriate than gross grasp measurements. Given the variation in grip strength across a population, the utility of these tools would be better assessed in a longitudinal fashion using a change in an individual subject's grip strength along the timeline of disease to assess the sensitivity of a tool in detecting functional reduction with time. Comparing the utility of cylindrical grasp with other tools used to assess hand function, such as pinch strength, also will be integral in better understanding hand function during early disease. Identifying an accurate method of quantifying functional decline of the thumb before extensive disease progression potentially provides a tool for clinically monitoring progression of thumb CMC OA, with an ultimate goal of improving thumb and hand function. Although the strength of association is important to consider when evaluating clinical tools, further research should be done to investigate the correlation of individual subjects' grip strength, as measured by gross and cylindrical grasp, with time to better understand the strength of the relationship between these tools and disease progression.

Acknowledgments We thank Tarpit Patel MS, (Bioengineering Laboratory, Department of Orthopaedics, Brown University, Providence, RI, USA) and Alexander Sox-Harris PhD, (Department of Orthopaedics, Stanford University, Stanford, CA, USA) for their invaluable contributions to our work.

\section{References}

1. Bagis S, Sahin G, Yapici Y, Cimen OB, Erdogan C. The effect of hand osteoarthritis on grip and pinch strength and hand function in postmenopausal women. Clin Rheumatol. 2003;22:420-424.

2. Cha SM, Shin HD, Kim KC, Park JW. Comparison of grip strength among 6 grip methods. J Hand Surg Am. 2014;39:22772284.

3. Chang JH, Ho KY, Su FC. Kinetic analysis of the thumb in jaropening activity among female adults. Ergonomics. 2008;51:843-857.

4. Cooney WP, Chao EY. Biomechanical analysis of static forces in the thumb during hand function. J Bone Joint Surg Am. 1977;59:27-36.

5. Dominick KL, Jordan JM, Renner JB, Kraus VB. Relationship of radiographic and clinical variables to pinch and grip strength among individuals with osteoarthritis. Arthritis Rheum. 2005;52:1424-1430.

6. Feix T, Romero J, Schmiedmayer HB, Dollar AM, Kragic D. The GRASP taxonomy of human grasp types. IEEE Trans Hum-Mach Syst. 2016;46:66-77.

7. Halilaj E, Moore DC, Laidlaw DH, Got CJ, Weiss AP, Ladd AL, Crisco JJ. The morphology of the thumb carpometacarpal joint does not differ between men and women, but changes with aging and early osteoarthritis. J Biomech. 2014;47:2709-2714.

8. Halilaj E, Moore DC, Patel TK, Ladd AL, Weiss A-PC, Crisco JJ. Early osteoarthritis of the trapeziometacarpal joint is not associated with joint instability during typical isometric loading. $J$ Orthop Res. 2015;33:1639-1645.
9. Halilaj E, Rainbow MJ, Got C, Schwartz JB, Moore DC, Weiss AP, Ladd AL, Crisco JJ. In vivo kinematics of the thumb carpometacarpal joint during three isometric functional tasks. Clin Orthop Relat Res. 2014;472:1114-1122.

10. Jansen CW, Niebuhr BR, Coussirat DJ, Hawthorne D, Moreno L, Phillip M. Hand force of men and women over 65 years of age as measured by maximum pinch and grip force. J Aging Phys Act. $2008 ; 16: 24-41$.

11. Jones G, Cooley HM, Bellamy N. A cross-sectional study of the association between Heberden's nodes, radiographic osteoarthritis of the hands, grip strength, disability and pain. Osteoarthritis Cartilage. 2001;9:606-611.

12. Kjeken I, Dagfinrud H, Slatkowsky-Christensen B, Mowinckel P, Uhlig T, Kvien TK, Finset A. Activity limitations and participation restrictions in women with hand osteoarthritis: patients' descriptions and associations between dimensions of functioning. Ann Rheum Dis. 2005;64:1633-1638.

13. Kodama R, Muraki S, Oka H, Iidaka T, Teraguchi M, Kagotani R, Asai Y, Yoshida M, Morizaki Y, Tanaka S, Kawaguchi H, Nakamura K, Akune T, Yoshimura N. Prevalence of hand osteoarthritis and its relationship to hand pain and grip strength in Japan: the third survey of the ROAD study. Mod Rheumatol. 2016;26:767-773.

14. Ladd AL, Messana JM, Berger AJ, Weiss AP. Correlation of clinical disease severity to radiographic thumb osteoarthritis index. J Hand Surg Am. 2015;40:474-482.

15. Lee AT, Williams AA, Lee J, Cheng R, Lindsey DP, Ladd AL. Trapezium trabecular morphology in carpometacarpal arthritis. $J$ Hand Surg Am. 2013;38:309-315.

16. Luker KR, Aguinaldo A, Kenney D, Cahill-Rowley K, Ladd AL. Functional task kinematics of the thumb carpometacarpal joint. Clin Orthop Relat Res. 2014;472:1123-1129.

17. Mathiowetz V, Kashman N, Volland G, Weber K, Dowe M, Rogers S. Grip and pinch strength: normative data for adults. Arch Phys Med Rehabil. 1985;66:69-74.

18. McQuillan TJ, Kenney D, Crisco JJ, Weiss AP, Ladd AL. Weaker functional pinch strength is associated with early thumb carpometacarpal osteoarthritis. Clin Orthop Relat Res. 2016;474:557-561.

19. Nunes PM, de Oliveira DG, Aruin AS, dos Santos MJ. Relationship between hand function and grip force control in women with hand osteoarthritis. J Rehabil Res Dev. 2012;49:855-865.

20. Puh U. Age-related and sex-related differences in hand and pinch grip strength in adults. Int J Rehabil Res. 2010;33:4-11.

21. Shechtman O, Sindhu BS. American Society of Hand Therapists Clinical Assessment Recommendations. 3rd ed. Mount Laurel, NJ: American Society of Hand Therapists; 2013. Available at: https:// www.asht.org/practice/clinical-assessment-recommendations. Accessed June 27, 2016.

22. Van Heest AE, Kallemeier P. Thumb carpal metacarpal arthritis. J Am Acad Orthop Surg. 2008;16:140-151.

23. Vergara M, Sancho-Bru JL, Gracia-Ibáñez V, Pérez-González A. An introductory study of common grasps used by adults during performance of activities of daily living. $J$ Hand Ther. 2014;27:225-233.

24. Villafañe $\mathrm{JH}$, Valdes K. Combined thumb abduction and index finger extension strength: a comparison of older adults with and without thumb carpometacarpal osteoarthritis. $J$ Manipulative Physiol Ther. 2013;36:238-244.

25. Villafañe JH, Valdes K, Bertozzi L, Negrini S. Minimal clinically important difference of grip and pinch strength in women with thumb carpometacarpal osteoarthritis when compared to healthy subjects. Rehabil Nurs. 2014 Dec 31; doi:10.1002/rnj.196. [Epub ahead of print].

26. Villafañe JH, Valdes K, Vanti C, Pillastrini P, Borboni A. Reliability of handgrip strength test in elderly subjects with unilateral 
thumb carpometacarpal osteoarthritis. Hand (N Y). 2015;10:205209.

27. Wimer B, Dong RG, Welcome DE, Warren C, McDowell TW. Development of a new dynamometer for measuring grip strength applied on a cylindrical handle. Med Eng Phys. 2009;31:695704.
28. Zhang Y, Niu J, Kelly-Hayes M, Chaisson CE, Aliabadi P, Felson DT. Prevalence of symptomatic hand osteoarthritis and its impact on functional status among the elderly: the Framingham study. Am J Epidemiol. 2002;156:1021-1027. 